\title{
LOCAL PROPERTIES OF QUOTIENT ANALYTIC SPACES
}

\author{
KUNIO TAKIJIMA AND TADASHI TOMARU
}

\begin{abstract}
Let $T=\mathbf{C} / \mathbf{Z}_{\omega_{1}}+\mathrm{Z}_{\omega_{2}}$ be a complex 1-torus and $E_{n}$ the set of all elliptic functions of order $n$. Then M. Namba showed that $E_{n}$ is a $2 n$-dimensional complex manifold. Let Aut $T$ be the automorphism group of $T$, then Aut $T$ is a 1-dimensional compact complex Lie group and the orbit space $E_{n} /$ Aut $T$ is an analytic space. In this paper, we shall show that $E_{n} /$ Aut $T$ has only rational singularities and if $n>5, E_{n} /$ Aut $T$ is rigid.
\end{abstract}

1. Introduction. Let $M$ be a complex manifold and $G$ a properly discontinuous transformation group on $M$. Then $\mathrm{H}$. Cartan [2] showed that the quotient space $M / G$ is a normal analytic space and D. Burns [1] has proved that $M / G$ is rational. Moreover $M$. Schlessinger [7], [8] showed that if $\operatorname{codim} F(G) \geqslant 3, M / G$ has only rigid singularities, where

$$
F(G):=\bigcup_{g \in G-\{e\}} F(g), F(g):=\{x \in M ; g x=x\}
$$

is the set of all fixed points.

In this paper, let $M$ be also a complex manifold and $G$ a complex Lie transformation group whose action is proper on $M$. Then H. Holmann [4] proved that the quotient space $M / G$ is a normal analytic space. We shall show the following

THEOREM 1. $M / G$ has only rational singularities. Moreover if codim $S(M / G) \geqslant 3, M / G$ is rigid, where $S(M / G)$ is the set of all singular points of $M / G$.

Let $\omega_{1}, \omega_{2} \in \mathbf{C} ; \operatorname{Im}\left(\omega_{2} / \omega_{1}\right)>0, T:=\mathbf{C} / \mathbf{Z} \omega_{1}+\mathbf{Z} \omega_{2}$ be a complex 1-torus and $E_{n}$ the set of all elliptic functions of order $n$. Then $E_{n}$ is a $2 n$-dimensional complex manifold [5] and the automorphism group Aut $T$ acts on $E_{n}$ naturally. Then we have

THEOREM 2. $E_{n} /$ Aut $T$ is rational and if $n \geqslant 5, E_{n} /$ Aut $T$ has only rigid singularities.

2. The proof of Theorem 1. We shall give some definitions. Let $\left(X, \theta_{X}\right)$ be an analytic space and $\pi:\left(\tilde{X}, \theta_{\tilde{X}}\right) \rightarrow\left(X, \theta_{X}\right)$ a resolution of singularities of $X$. Then $x \in X$ is called a rational singularity if $\left(R^{i} \pi_{*} \theta_{\tilde{X}}\right)_{x}=0$ for any $i>0$

Received by the editors December 13, 1977 and, in revised form, March 27, 1978.

AMS (MOS) subject classifications (1970). Primary 32M05, 32C40.

Key words and phrases. Quotient analytic space, rational singularity, rigid singularity, elliptic function.

๑ American Mathematical Society 1978 
(cf. [1]). $x \in X$ is called a rigid singularity if any local flat deformation of $(X, x)$ is locally trivial (cf. [7], [8]).

Let $R$ be an equivalence relation on $X$. We identify the graph of $R$ with $R$. Then $R$ is called open (resp. proper, finite) if the natural projection $p_{1}: R \rightarrow X$ is open (resp. proper, finite). And $R$ is called analytic if the graph $R$ is an analytic set in $X \times X$ (cf. [4], [9]).

Let $M$ be a complex manifold and $G$ a complex Lie transformation group on $M$. Then $G$ is called proper on $M$ if the graph mapping $\Psi: G \times M \rightarrow M$ $\times M, \Psi(g, x):=(g x, x)$ is proper. (This is different from the above definition that an equivalence relation $R$ is proper.) Let $G(x):=\{g x$; $g \in G\}$ be the orbit through $x$ and $G_{x}:=\{g \in G ; g x=x\}$ an isotropy group at $x$.

We shall prove Theorem 1 and its proof is essentially owing to the technique by H. Holmann [3], [4]. Let $\Phi: G \times M \rightarrow M, \Phi(g, x):=g x$ be the natural holomorphic mapping. Then $G(x)=\Phi(G \times\{x\})$ is a complex submanifold of $M$ and biholomorphic to $G / G_{x}$. Since $G$ is proper on $M$, $M / G$ is Hausdorff with respect to the quotient topology. Hence from Hilfssatz 7 of [4], for any $x \in M$ there exist an open connected neighborhood $U \subset M$ of $x$ and a submanifold $N \subset U$ such that

(1) $x \in N$,

(2) $U \cong(G(x) \cap U) \times N$,

(3) $G(y) \cap U \cong(G(x) \cap U) \times N_{y}$ for any $y \in N$, where $N_{y}:=G(y) \cap$ $N$ is finite and $N_{x}=\{x\}$. Let $R$ be an equivalence relation on $N$ defined by $R(y):=N_{y}$ for any $y \in N$. Then $R$ is open proper finite analytic (if we choose $U$ small enough) and $M / G$ is locally isomorphic to $N / R$ (cf. the proof of Satz 15 in [4]).

For any $g \in G_{x}$, there exists an open connected neighborhood $V \subset N$ of $x$ such that $g^{\prime}:=q g: V \rightarrow g^{\prime}(V) \subset N$ is biholomorphic, where $q: U \rightarrow N$ is the natural projection and the inverse of $g^{\prime}$ is

$$
\left(g^{\prime}\right)^{-1}:=q g^{-1} \text {. }
$$

Let $G^{\prime}(V)$ be the set of all biholomorphic mappings on $V$ induced from $G_{x}$ for any connected neighborhood $V \subset N$ of $x$. Since $G$ is proper on $M$, there exists an open connected neighborhood $V \subset N$ of $x$ such that $G^{\prime}(V)$ is a finite transformation group on $V$ and $R$ coincides with the equivalence relation induced by $G^{\prime}(V)$ on $V$ (cf. the proof of Satz 19 in [4]). Therefore $M / G$ is locally isomorphic to $V / G^{\prime}(V)$ and so $M / G$ has only rational singularities. If we show the following lemma, the proof of Theorem 1 is complete.

LEMMA 1. Let $\Gamma$ be a finite subgroup of $\operatorname{GL}(n, \mathrm{C})$. If $\operatorname{codim} S\left(\mathbf{C}^{n} / \Gamma\right) \geqslant 3$, $\mathrm{C}^{n} / \Gamma$ is rigid.

Proof. Let $\Gamma_{0}$ be the normal subgroup of $\Gamma$ generated by reflections, where $g \in \operatorname{GL}(n, \mathbf{C})$ is called a reflection if $g$ is order-finite and $\operatorname{codim} F(g)=1$. 
Then $\mathbf{C}^{n} / \Gamma_{0}$ is biholomorphic to $\mathbf{C}^{n}$ and $\Gamma / \Gamma_{0}$ acts on $\mathbf{C}^{n} / \Gamma_{0}$ naturally. Moreover $\mathbf{C}^{n} / \Gamma \cong\left(\mathbf{C}^{n} / \Gamma_{0}\right) /\left(\Gamma / \Gamma_{0}\right)$ and $\Gamma / \Gamma_{0}$ has no reflection (cf. [6]). Thus we may assume that $\Gamma$ has no reflection. Then $p(F(\Gamma))=S\left(C^{n} / \Gamma\right)$, where $p$ : $\mathbf{C}^{n} \rightarrow \mathbf{C}^{n} / \Gamma$ is the projection.

In fact, $p(F(\Gamma)) \supset S\left(\mathbf{C}^{n} / \Gamma\right)$ is clear. Let $x \in \mathbf{C}^{n}$ and $p(x) \notin S\left(\mathbf{C}^{n} / \Gamma\right)$, then there exists a nonsingular neighborhood $U \subset \mathbf{C}^{n} / \Gamma$ of $p(x)$ such that $p$ : $p^{-1}(U) \rightarrow U$ is a finite covering between complex manifolds. We have

$$
F(\Gamma) \cap p^{-1}(U)=\left\{t \in p^{-1}(U) ; J p(t)=0\right\},
$$

where $J p$ is the Jacobian of $p$. Since $\operatorname{codim} F(\Gamma) \geqslant 2, F(\Gamma) \cap p^{-1}(U)$ must be empty. Thus $x \notin F(\Gamma)$. Therefore $\operatorname{codim} F(\Gamma)=\operatorname{codim} S\left(\mathbf{C}^{n} / \Gamma\right) \geqslant 3$ and so $\mathbf{C}^{n} / \Gamma$ is rigid.

ReMARK. If $G$ is not proper on $M$, locally $M / G$ is not always isomorphic to $V / G^{\prime}(V)$.

3. The proof of Theorem 2. Since Aut $T$ is compact, Aut $T$ is proper on $E_{n}$. Hence the first part of Theorem 2 is straightforward from Theorem 1. Thus we must calculate codim $S\left(E_{n} /\right.$ Aut $\left.T\right)$.

Let $S_{n}$ be the $n$-symmetric group. Then $S_{n} \times S_{n}$ acts on $\mathrm{C}^{2 n+1}$ as follows:

$$
\begin{gathered}
\left(S_{n} \times S_{n}\right) \times \mathbf{C}^{2 n+1} \rightarrow \mathbf{C}^{2 n+1}, \\
\left(g_{1}, g_{2}, \alpha, \beta, \gamma\right) \leadsto\left(g_{1} \alpha, g_{2} \beta, \gamma\right),
\end{gathered}
$$

where $\alpha=\left(\alpha_{1}, \ldots, \alpha_{n}\right), \beta=\left(\beta_{1}, \ldots, \beta_{n}\right)$. We define

$$
\begin{gathered}
\phi: \mathbf{C}^{2 n+1} \rightarrow \mathbf{C}^{2 n+1}, \\
\phi(\alpha, \beta, \gamma):=\left(\sigma_{1}(\alpha), \ldots, \sigma_{n}(\alpha), \sigma_{1}(\beta), \ldots, \sigma_{n}(\beta), \gamma\right),
\end{gathered}
$$

where $\sigma_{i}$ is the $i$-fundamental symmetric function $(i=1, \ldots, n)$. Then we have $\mathbf{C}^{2 n+1} /\left(S_{n} \times S_{n}\right) \cong \phi\left(\mathbf{C}^{2 n+1}\right)=\mathbf{C}^{2 n+1}$.

For $\omega_{1}, \omega_{2} \in \mathbf{C} ; \operatorname{Im}\left(\omega_{2} / \omega_{1}\right)>0$, we put $\Delta:=\mathbf{Z} \omega_{1}+\mathbf{Z} \omega_{2}, \Delta^{*}:=\Delta-\{0\}$ and

$$
\begin{aligned}
\tilde{S}_{\Omega}:=\left\{(\alpha, \beta, \gamma) \in \mathbf{C}^{2 n+1} ; \sum_{i=1}^{n} \alpha_{i}-\sum_{i=1}^{n} \beta_{i}=\Omega, \gamma \neq 0, \alpha_{i}-\beta_{j} \notin \Delta,\right. \\
\left.\alpha_{i}-\alpha_{j} \notin \Delta^{*}, \beta_{i}-\beta_{j} \notin \Delta^{*}(i, j=1, \ldots, n)\right\}
\end{aligned}
$$

for $\Omega \in \Delta$. Since $\tilde{S}_{\Omega}$ is an $S_{n} \times S_{n}$-invariant complex manifold,

$$
\begin{aligned}
S_{\Omega}:=\phi\left(\tilde{S}_{\Omega}\right)=\left\{(x, y, z) \in \mathbf{C}^{2 n+1} ; x_{1}-y_{1}=\Omega, z \neq 0, \alpha_{i}-\beta_{j} \notin \Delta,\right. \\
\left.\alpha_{i}-\alpha_{j} \notin \Delta^{*}, \beta_{i}-\beta_{j} \notin \Delta^{*}(i, j=1, \ldots, n)\right\}
\end{aligned}
$$

is also a $2 n$-dimensional complex manifold, where $\alpha_{i}, \beta_{i}$ are roots of

$$
\begin{gathered}
X^{n}-x_{1} X^{n-1}+x_{2} X^{n-2}+\cdots+(-1)^{n} x_{n}=0, \\
X^{n}-y_{1} X^{n-1}+y_{2} X^{n-2}+\cdots+(-1)^{n} y_{n}=0
\end{gathered}
$$

respectively. And $\tilde{S}:=\cup_{\Omega \in \Delta} \tilde{S}_{\Omega}, S:=\cup_{\Omega \in \Delta} S_{\Omega}$ are complex manifolds. 
By the classical theory of elliptic functions, for any $f(w) \in E_{n}$, let $\alpha_{1}, \ldots, \alpha_{n}, \beta_{1}, \ldots, \beta_{n}$ be zeroes and poles of $f(w)$ respectively, then

$$
\begin{gathered}
\sum_{i=1}^{n} \alpha_{i}-\sum_{i=1}^{n} \beta_{i}=\Omega \in \Delta, \quad \Omega=p_{0} \omega_{1}+q_{0} \omega_{2}, \\
f(w)=\gamma \exp 2\left(p_{0} \eta_{1}+q_{0} \eta_{2}\right) w \prod_{i=1}^{n} \frac{\sigma\left(w-\alpha_{i}\right)}{\sigma\left(w-\beta_{i}\right)},
\end{gathered}
$$

where $\eta_{j}:=\zeta\left(\frac{1}{2} \omega_{j}\right)(j=1,2)$ and $\sigma, \zeta$ are the Weierstrass functions. We define $\tilde{F}: \tilde{S} \rightarrow E_{n}$

$$
\tilde{F}(\alpha, \beta, \gamma):=\gamma \exp 2\left(p_{0} \eta_{1}+q_{0} \eta_{2}\right) w \prod_{i=1}^{n} \frac{\sigma\left(w-\alpha_{i}\right)}{\sigma\left(w-\beta_{i}\right)},
$$

where

$$
\sum_{i=1}^{n} \alpha_{i}-\sum_{i=1}^{n} \beta_{i}=p_{0} \omega_{1}+q_{0} \omega_{2} \in \Delta
$$

Since $\tilde{F}$ is $S_{n} \times S_{n}$-invariant, $\tilde{F}: \tilde{S} \rightarrow E_{n}$ induces $F: S \rightarrow E_{n}$

$$
F(x, y, z):=z \exp 2\left(p_{0} \eta_{1}+q_{0} \eta_{2}\right) w \prod_{i=1}^{n} \frac{\sigma\left(w-\alpha_{i}\right)}{\sigma\left(w-\beta_{i}\right)},
$$

where $x_{1}-y_{1}=p_{0} \omega_{1}+q_{0} \omega_{2} \in \Delta$ and $\alpha_{i}, \beta_{i}$ are roots of (1), (2) respectively. Then we can show the following lemma (cf. [5, Remark, p. 75]).

LEMMA 2. $F: S \rightarrow E_{n}$ is surjective open holomorphic and locally biholomorphic.

We shall calculate $\operatorname{codim} S\left(E_{n} /\right.$ Aut $\left.T\right)$ using this locally biholomorphic mapping $F$. Here we assume that $\omega_{2} / \omega_{1} \npreceq e^{\pi i / 2}, e^{2 \pi i / 3}$ under the modular group. Then Aut $T$ has two connected components $T_{0}, T_{1}$ and $T_{0}$ is isomorphic to $T$ as Lie group. We put

$$
\begin{gathered}
g_{1}\left(x_{1}, x_{2}, x_{3}, n\right):=-x_{3}+\frac{n-2}{n} x_{1} x_{2}-\frac{(n-1)(n-2)}{3 n^{2}} x_{1}^{3}, \\
g_{2}\left(x_{1}, x_{2}, x_{3}, x_{4}, x_{5}, n\right):=-x_{5}+\frac{n-4}{n} x_{1} x_{4}-\frac{(n-3)(n-4)}{n^{2}} x_{1}^{2} x_{3} \\
+\frac{2(n-2)(n-3)(n-4)}{3 n^{3}} x_{1}^{3} x_{2}-\frac{(n-1)(n-2)(n-3)(n-4)}{5 n^{4}} x_{1}^{5}, \\
\rho(x, \lambda):=\lambda^{n}+x_{1} \lambda^{n-1}+\cdots+x_{n-1} \lambda+x_{n}, x=\left(x_{1}, \ldots, x_{n}\right), \\
a_{i}(x, \lambda):=\frac{1}{(n-i) !} \rho^{(n-i)}(x, \lambda) \\
=\left(\begin{array}{c}
n \\
i
\end{array}\right) \lambda^{i}+\left(\begin{array}{c}
n-1 \\
i-1
\end{array}\right) x_{1} \lambda^{i-1}+\cdots+(n-(i-1)) x_{i-1} \lambda+x_{i}, \\
a_{i}^{\prime}(x, \lambda):=(-1)^{i} a_{i}(x, \lambda), \quad t:=-\left(\lambda+2 x_{1} / n\right) .
\end{gathered}
$$


Then we have the following lemma by long elementary calculation.

LEMMA 3.

$$
\begin{gathered}
a_{1}^{\prime}(x, \lambda)=a_{1}(x, t), a_{2}^{\prime}(x, \lambda)=a_{2}(x, t), \\
a_{3}^{\prime}(x, \lambda)=a_{3}(x, t)+2 g_{1}\left(x_{1}, x_{2}, x_{3}, n\right), \\
a_{4}^{\prime}(x, \lambda)=a_{4}(x, t)+2(n-3)\left(t+x_{1} / n\right) g_{1}\left(x_{1}, x_{2}, x_{3}, n\right), \\
a_{5}^{\prime}(x, \lambda)=a_{5}(x, t)+(n-3)(n-4)\left(t^{2}+2 x_{1} t / n\right) g_{1}\left(x_{1}, x_{2}, x_{3}, n\right) \\
+2 g_{2}\left(x_{1}, x_{2}, x_{3}, x_{4}, x_{5}, n\right), \\
a_{6}^{\prime}(x, \lambda)= \\
a_{6}(x, t)+(n-3)(n-4)\left(\frac{n-5}{3} t^{3}+\frac{n-5}{n} x_{1} t^{2}-\frac{1}{3 n^{2}} x_{1}^{2}\right) g_{1} \\
\quad \cdot\left(x_{1}, x_{2}, x_{3}, n\right)+2(n-5)\left(t+x_{1} / n\right) g_{2}\left(x_{1}, x_{2}, x_{3}, x_{4}, x_{5}, n\right) .
\end{gathered}
$$

In general,

$$
\begin{array}{r}
a_{i}^{\prime}(x, \lambda)=a_{i}(x, t)+\sum_{j=0}^{i-3}\left(\sum_{k=0}^{i-j}(-1)^{k}\left(\begin{array}{c}
n-k \\
i-k
\end{array}\right)\left(\begin{array}{c}
i-k \\
i-j-k
\end{array}\right)\left(\frac{2}{n} x_{1}\right)^{i-j-k}\right. \\
\left.\cdot x_{k}-\left(\begin{array}{c}
n-i+j \\
j
\end{array}\right) x_{i-j}\right) t^{j} \\
(i=1, \ldots, n) .
\end{array}
$$

Let

$$
\begin{aligned}
A_{n}:=\left\{(x, y, z) \in S_{0} ;\right. & a_{i}^{\prime}\left(x,-2 x_{1} / n\right)=x_{i} \\
a_{i}^{\prime}\left(y,-2 y_{1} / n\right) & \left.=y_{i}(i=1, \ldots, n)\right\} .
\end{aligned}
$$

(If $i=1,2, a_{i}^{\prime}\left(x,-2 x_{1} / n\right)=x_{i}$ and $a_{i}^{\prime}\left(y,-2 y_{1} / n\right)=y_{i}$ are always true.) Then $A_{n}=\left\{(x, y, z) \in S_{0} ; g_{1}\left(x_{1}, x_{2}, x_{3}, n\right)=g_{1}\left(y_{1}, y_{2}, y_{3}, n\right)=0\right\}$ for $n=3$, 4 and $A_{n}=\left\{(x, y, z) \in S_{0} ; g_{1}\left(x_{1}, x_{2}, x_{3}, n\right)=g_{2}\left(x_{1}, x_{2}, x_{3}, x_{4}, x_{5}, n\right)=g_{1}\left(y_{1}\right.\right.$, $\left.\left.y_{2}, y_{3}, n\right)=g_{2}\left(y_{1}, y_{2}, y_{3}, y_{4}, y_{5}, n\right)=0\right\}$ for $n=5$, 6. Thus if $n \geqslant 5$, we have $\operatorname{codim} A_{n} \geqslant 4$.

Now we shall prove the rest of Theorem 2. For any $f(w) \in E_{n}$, there exists $(\alpha, \beta, \gamma) \in \tilde{S}_{\Omega}$ such that

$$
f(w)=\gamma \exp 2\left(p_{0} \eta_{1}+q_{0} \eta_{2}\right) w \prod_{i=1}^{n} \frac{\sigma\left(w-\alpha_{i}\right)}{\sigma\left(w-\beta_{i}\right)}, \quad \Omega=p_{0} \omega_{1}+q_{0} \omega_{2} .
$$

Let $B_{n}:=\{(x, y, z) \in S$; (1) and (2) have no simple root $\}$, then $B_{n}$ is an analytic set in $S$. If $(a, b, \gamma):=\phi(\alpha, \beta, \gamma) \in\left(S-B_{n}\right) \cup S_{0}$, we may assume that $(a, b, \gamma) \in S_{0}$. Then 


$$
f(w)=\gamma \prod_{i=1}^{n} \frac{\sigma\left(w-\alpha_{i}\right)}{\sigma\left(w-\beta_{i}\right)}, \quad a_{1}=b_{1} .
$$

For any $a(w) \in T_{0} ; a(w)=w-\lambda$, we have

$$
f \circ a(w)=\gamma \prod_{i=1}^{n} \frac{\sigma\left(w-\lambda-\alpha_{i}\right)}{\sigma\left(w-\lambda-\beta_{i}\right)} .
$$

Hence $(\lambda+\alpha, \lambda+\beta, \gamma) \in \tilde{S}_{0} \cap \tilde{F}^{-1}(f \circ a(w))$ and $\phi(\lambda+\alpha, \lambda+\beta, \gamma)=$ $\left(a_{1}(a, \lambda), \ldots, a_{n}(a, \lambda), a_{1}(b, \lambda), \ldots, a_{n}(b, \lambda), \gamma\right) \in S_{0} \cap F^{-1}(f \circ a(w))$. For any $a(w) \in T_{1} ; a(w)=-w-\lambda$, we have

$$
f \circ a(w)=\gamma \prod_{i=1}^{n} \frac{\sigma\left(w+\lambda+\alpha_{i}\right)}{\sigma\left(w+\lambda+\beta_{i}\right)} .
$$

Hence $(-\lambda-\alpha,-\lambda-\beta, \gamma) \in \tilde{S}_{0} \cap \tilde{F}^{-1}(f \circ a(w))$ and $\phi(-\lambda-\alpha$, $-\lambda-\beta, \gamma)=\left(a_{1}^{\prime}(a, \lambda), \ldots, a_{n}^{\prime}(a, \lambda), a_{1}^{\prime}(b, \lambda), \ldots, a_{n}^{\prime}(b, \lambda), \gamma\right) \in S_{0} \cap$ $F^{-1}(f \circ a(w))$. Namely the orbit (Aut $\left.T\right)(f(w))$ is locally described by $\left\{\left(a_{1}(a, \lambda), \ldots, a_{n}(a, \lambda), a_{1}(b, \lambda), \ldots, a_{n}(b, \lambda), \gamma\right) ; \lambda \in T\right\} \cup$ $\left\{\left(a_{1}^{\prime}(a, \lambda), \ldots, a_{n}^{\prime}(a, \lambda), a_{1}^{\prime}(b, \lambda), \ldots, a_{n}^{\prime}(b, \lambda), \gamma\right) ; \lambda \in T\right\}$ in $S_{0}$. Hence using Lemma 3, we can show that (Aut $T)_{f(w)}=\{w\}$ if $(a, b, \gamma) \notin A_{n}$. Then $E_{n} /$ Aut $T$ is nonsingular at $p(f(w))$ by the proof of Theorem 1 . If $n \geqslant 5$, we have $\operatorname{codim} A_{n} \geqslant 4$ and $\operatorname{codim} B_{n} \geqslant n \geqslant 5$. Therefore $\operatorname{codim} S\left(E_{n} /\right.$ Aut $\left.T\right)$ $\geqslant 3$ and so $E_{n} /$ Aut $T$ is rigid.

In the case $\omega_{2} / \omega_{1} \cong e^{\pi i / 2}$ or $e^{2 \pi i / 3}$, we can also show that if $n \geqslant 5$, $\operatorname{codim} S\left(E_{n} /\right.$ Aut $\left.T\right) \geqslant 3$ by almost the same argument as above.

EXAMPLE. Let $(w)$ be the Weierstrass pe-function. Then

$$
\wp(w)^{2}=\frac{1}{\sigma(\alpha)^{4}} \frac{\sigma(w-\alpha)^{2} \sigma(w+\alpha)^{2}}{\sigma(w)^{4}} \in E_{4}(\wp(\alpha)=0)
$$

and (Aut $T)_{\wp(w)^{2}}=\{w,-w\}$. Thus $\left(\alpha, \alpha,-\alpha,-\alpha, 0,0,0,0,1 / \sigma(\alpha)^{4}\right) \in \tilde{S}_{0}$ $\cap \tilde{F}^{-1}\left(\wp(w)^{2}\right)$ and $\left(0,-2 \alpha^{2}, 0, \alpha^{4}, 0,0,0,0,1 / \sigma(\alpha)^{4}\right) \in S_{0} \cap F^{-1}\left(\wp(w)^{2}\right)$. By Lemma 3 and the proof of Theorems 1 and 2, we may take $N:=\left\{x_{1}=\right.$ $\left.y_{1}=0\right\} \subset S_{0}$ and for any $(x, y, z)=\left(0, x_{2}, x_{3}, x_{4}, 0, y_{2}, y_{3}, y_{4}, z\right) \in N$, the orbit (Aut $T)(F(x, y, z))$ is locally described in $S_{0}$ as follows:

$$
\begin{gathered}
\left\{\left(4 \lambda, 6 \lambda^{2}+x_{2}, 4 \lambda^{3}+2 x_{2} \lambda+x_{3}, \lambda^{4}+x_{2} \lambda^{2}+x_{3} \lambda+x_{4}, 4 \lambda, 6 \lambda^{2}+y_{2},\right.\right. \\
\left.\left.4 \lambda^{3}+2 y_{2} \lambda+y_{3}, \lambda^{4}+y_{2} \lambda^{2}+y_{3} \lambda+y_{4}, z\right) ; \lambda \in T\right\} \\
\cup\left\{\left(-4 \lambda, 6 \lambda^{2}+x_{2},-4 \lambda^{3}-2 x_{2} \lambda-x_{3}, \lambda^{4}+x_{2} \lambda^{2}+x_{3} \lambda+x_{4},-4 \lambda,\right.\right. \\
\left.\left.6 \lambda^{2}+y_{2},-4 \lambda^{3}-2 y_{2} \lambda-y_{3}, \lambda^{4}+y_{2} \lambda^{2}+y_{3} \lambda+y_{4}, z\right) ; \lambda \in T\right\} .
\end{gathered}
$$

Hence $N_{(x, y, z)}=\left\{\left(0, x_{2}, x_{3}, x_{4}, 0, y_{2}, y_{3}, y_{4}, z\right),\left(0, x_{2},-x_{3}, x_{4}, 0, y_{2},-y_{3}, y_{4}\right.\right.$, $z)\}$. Therefore $E_{4} /$ Aut $T$ is isomorphic to $\left\{w^{2}-u v=0\right\} \times \mathbf{C}^{5} \subset \mathbf{C}^{8}$ at $p$ $\left(\wp(w)^{2}\right)$ and so not rigid. 


\section{REFERENCES}

1. D. Burns, On rational singularities in dimension > 2, Math. Ann. 211 (1974), 237-244.

2. H. Cartan, Quotient d'un espace analytique par un groupe d'automorphismes, Algebraic Geometry and Topology. A symposium in honor of S. Lefschetz, Princeton Univ. Press, Princeton, N. J., 1957, pp. 90-102.

3. H. Holmann, Quotienten komplexer Räume, Math. Ann. 142 (1961), 407-440.

4. Komplexe Räume mit komplexen Transformationsgruppen, Math. Ann. 150 (1963), 327-360.

5. M. Namba, Moduli of open holomorphic maps of compact complex manifolds, Math. Ann. 220 (1976), 65-76.

6. D. Prill, Local classification of quotients of complex manifolds by discontinuous groups, Duke Math. J. 34 (1967), 375-386.

7. M. Schlessinger, Rigidity of quotient singularities, Invent. Math. 14 (1971), 17-26.

8. _ On rigid singularities, Proc. Conf. on Singularities, Rice Univ. Studies, Houston, Tex., 1972, pp. 147-162.

9. K. Takijima and T. Suzuki, On the trivial extension of equivalence relations on analytic spaces, Trans. Amer. Math. Soc. 219 (1976), 369-377.

Department of Mathematics, faculty of Education, Saitama University, Urawa, Japan 